\title{
Adhesion of a New Commercial Self-etching/Self-priming Bonding Resin to Human Caries-infected Dentin
}

\author{
Takeshi KIMOCHI, Masahiro YOSHIYAMA, Akihisa URAYAMA \\ and Takashi MATSUO \\ Department of Conservative Dentistry, School of Dentistry, \\ Tokushima University, 3-18-15, Kuramoto-cho, \\ Tokushima 770-8504, Japan
}

Received June 7, 1999/Accepted September 13, 1999

\begin{abstract}
We have examined the adhesive properties of a new commercial self-etching/self-priming bonding resin (Unifil Bond, UB) to normal and caries-infected dentin of human extracted molars using scanning electron microscopy (SEM) and a micto-tensile bonding strength (MTBS) test. In this study, 7 human extracted molars with moderate occlusal caries were used, and flat surfaces including occlusal dentin caries were prepared from the teeth. After the application of UB to the surfaces, a composite resin was built up, and subjected to the measurement of MTBS and SEM observation of the interfacial morphology between UB and dentin. The MTBS of UB to normal dentin was $33.4 \mathrm{MPa}$, but that to caries-infected dentin was $11.0 \mathrm{MPa}$. There was a significant difference between the MTBS to normal and carious dentin. SEM observation revealed that the typical hybrid layer was not formed in caries-infected dentin. These results suggested that resin infiltration into caries-infected dentin was not sufficient to allow perfect sealing of the restoration.
\end{abstract}

Key words : Resin, Adhesion, Caries-infected dentin

\section{INTRODUCTION}

Recent adhesive systems exhibit high bond strengths to human normal dentin, however, their adhesive properties to caries-infected dentin are still unclear. Although most bonding strength testing is performed using normal dentin, caries-affected dentin and sclerotic cervical dentin are often the clinically-relevant bonding substrates.

Recently, the micro-tensile bond strength (MTBS) test has been introduced to evaluate specimens using small areas $\left(0.5 \sim 4.0 \mathrm{~mm}^{2}\right)$ for bonding ${ }^{1,2}$. This new method tends to produce higher bond strengths than conventional methods, and produces primarily adhesive failures at bonded surface areas below $2 \mathrm{~mm}^{2}$, presumably because of more uniform stress distributions. Using this method, it is possible to measure the bond strengths of resins to caries-affected dentin and sclerotic dentin.

Nakajima et $a l_{.}{ }^{3)}$ used the MTBS test to compare bond strengths made to either caries-affected dentin or normal dentin by current bonding systems, and suggested that the strength of adhesion to dentin depends upon both the adhesive systems and the type of dentin. We have compared the regional bond strengths and SEM appearances of adhesive bonds made to occlusal vs. gingival surfaces of sclerotic dentin in 
natural wedge-shaped defects and in the same region in artificially prepared normal dentin, and reported that the MTBS values of current bonding systems to sclerotic dentin were significantly lower than those to prepared normal dentin ${ }^{4)}$.

Hybridized dentin permits dental treatments that were previously impossible with conventional techniques, opening new frontiers in modern adhesive dentistry ${ }^{5)}$. MertzFairhurst et al. suggested that dentin caries can be arrested with resin sealant without removal, and termed this caries treatment as sealed restoration ${ }^{6)}$. If the residual bacteria in caries-infected dentin can be embedded by adhesive resins, and if these embedded bacteria become inactive, dentin caries must be arrested.

The purpose of this study was to measure the MTBS of a new self-etching/selfpriming bonding resin (UB) to normal and carious dentin, and to observe the interfacial morphology of UB-bonded dentin by SEM.

\section{MATERIALS AND METHOD}

\section{Specimen preparation}

The components of the self-etching/self-priming bonding resin, Unifil Bond (UB, GC Co. Ltd., Tokyo, Japan), are shown in Table 1. UB-Primer is a one liquid type selfetching primer, and contains 4-MET (4-methacryloxyethyl trimellitate) as an adhesive monomer. The UB bonding agent has UDMA (urethan dimethacrylate) as its base monomer.

Seven freshly extracted human molars with coronal medium carious dentin were used in this study, and 3 teeth were subjected to MTBS, 2 teeth to SEM of the adhesive interfaces, and 2 teeth to SEM of the effect of the primer. The occlusal surface was ground perpendicular to the long axis of the tooth to expose a flat surface where the caries-infected lesion was surrounded by normal dentin as shown in Fig. 1.

In order to obtain carious dentin, grinding was performed under running water using the combined criteria of visual examination and staining with a caries detector solution (Kuraray Co. Ltd., Osaka, Japan) as slightly-pink area. The flat prepared dentin surfaces were polished with \#600 silicon carbide paper and UB-primer was applied to the polished dentin surface for $20 \mathrm{sec}$. After gentle air drying for $5 \mathrm{sec}$, the UB bonding agent was applied on the surface, and light-cured for $10 \mathrm{sec}$. A composite resin (Clearfil AP-X, Kuraray Co. Ltd., Osaka, Japan) was then built up to a height of about $5 \mathrm{~mm}$. The resin-bonded teeth were then stored in water $\left(37^{\circ} \mathrm{C}\right)$ for $24 \mathrm{~h}$, and subjected to the MTBS test and SEM observation.

Table 1 The composition of Unifil Bond (UB, GC)

\begin{tabular}{lcc}
\hline & (Batch No.) & Composition \\
\hline Primer & $(100781)$ & 4-MET, HEMA, Ethanol, Distilled water \\
Bonding agent & $(090781)$ & UDMA, HEMA, TEGDMA, CQ \\
\hline
\end{tabular}

$\mathrm{HEMA}=$ hydroxyethylmethacrylate, TEGDMA = triethyleneglycol dimethacrylate, $\mathrm{CQ}=$ camphorquinone 


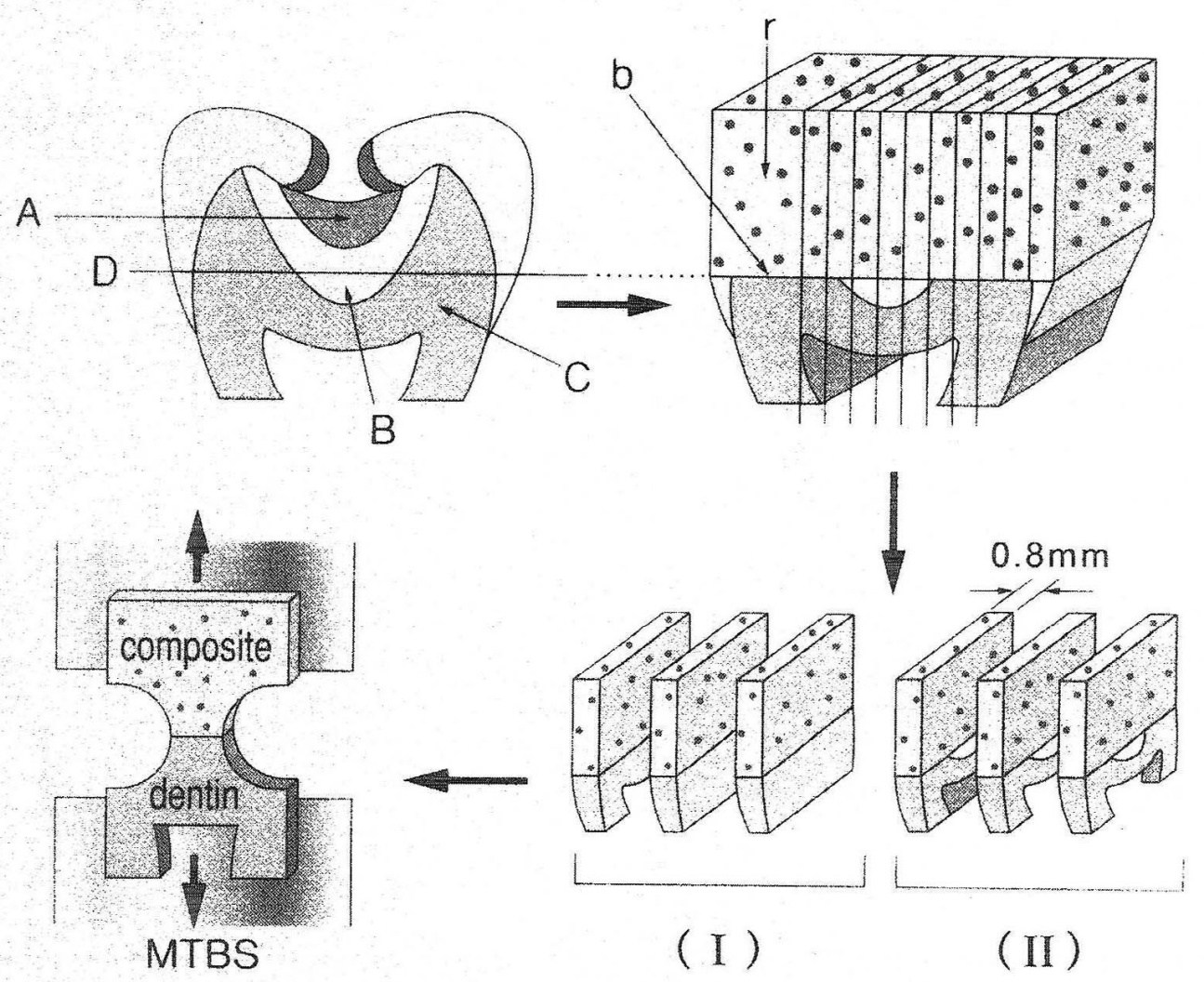

Fig. 1 Schematic showing how carious teeth were prepared for bonding and for MTBS testing.

A: softened dentin, B: caries-infected dentin (slightly-pink area), C: normal dentin, D: ground surface, b: bonding layer, r: composite resin, ( I ): normal dentin adhesive specimen, ( II ): caries-infected dentin adhesive specimens.

\section{MTBS measurement}

Three of the resin-bonded teeth were subjected to MTBS measurement. As shown in Fig. 1, 5 or 6 resin-bonded dentin slices $(0.8 \mathrm{~mm}$ thick $)$ were made from each tooth, parallel to the long axis of the tooth using a low-speed diamond saw (Isomet, Buehler Co. Ltd., Lake Bluff, IL, USA). The resin-bonded dentin slices were trimmed to yield a $1 \mathrm{~mm}^{2}$ cross-sectional area with an ultra-fine diamond bur in a high-speed handpiece under water-cooling. Eight normal dentin adhesive specimens and 6 caries-infected dentin adhesive specimens were obtained. The specimens were attached to a Bencor Multi-T testing apparatus (Danville Engineering Co., Danville, CA, USA) with a cyanoacrylate adhesive, and subjected to tensile stress in a universal testing machine (Autograph AGS-D, Shimadzu Co. Ltd, Kyoto, Japan) at a cross-head speed of 1 
$\mathrm{mm} / \mathrm{min}$. The results of the MTBS testing were statistically analyzed with the Student's t-test.

\section{SEM observation of the adhesive interfaces}

Two of the resin-bonded teeth were subjected to SEM observation. The resin-bonded teeth were sectioned into two halves parallel to the long axes of the teeth using a low-speed diamond saw. The cut surfaces were ground with silicon carbide abrasive papers, highly polished with a diamond paste, and then immersed into $0.5 \mathrm{~N} \mathrm{HCl} \mathrm{solu-}$ tion for $15 \mathrm{sec}$. Following $15 \mathrm{sec}$ rinsing with water, the specimens were subjected to 2 min treatment with $5 \% \mathrm{NaOCl}$ solution. The specimens were rinsed thoroughly with water, fixed in $2 \%$ glutaraldehyde solution for $2 \mathrm{~h}$, dehydrated through an ascending series of ethanol solutions and critical-point dried. They were then examined under an SEM (Hitachi S-700, Hitachi Co. Ltd., Tokyo, Japan) at $20 \mathrm{kV}$ accelerating voltage.

\section{SEM of the effect of UB-primer to normal and carious dentin}

Flat dentin surfaces were prepared on two of the extracted carious teeth as described previously. UB-primer was applied to the flat surface including carious dentin for 20 sec, and dried for $5 \mathrm{sec}$ with a gentle air blow. Then the flat dentin surfaces were subjected to SEM observation as described previously.

\section{RESULTS}

Table 2 indicates the results of MTBS of UB to normal and carious dentin. The MTBS of UB to normal dentin was $33.4 \mathrm{MPa}$, but that to carious dentin was 11.0 $\mathrm{MPa}$. There was a significant difference between the MTBS to normal dentin and carious dentin $(p<0.01)$. The most common fracture in normal dentin was adhesive fracture. However in carious dentin, cohesive fracture was the most common mode.

An SEM image of UB-bonded dentin is shown in Fig. 2. (A) shows the adhesive interface of UB to normal dentin. The hybrid layer formed by UB was very shallow (about $1-2 \mu \mathrm{m}$ thick), and there were short resin tags $(2-3 \mu \mathrm{m})$. (B) shows the adhesive interface of UB to carious dentin. There are mud-like structures and gap formation among the bonding agents and dentin. The formation of resin tags could not be

Table 2 MTBS of UB to normal vs. caries-infected dentin and failure modes

\begin{tabular}{lccc}
\hline \multirow{2}{*}{ Dentin } & MTBS $(\mathrm{MPa})(\mathrm{n})$ & & Failure mode \\
\cline { 2 - 2 } Normal & $($ Mean \pm S.D. $)$ & & Adhesive $(\mathrm{n}) /$ Cohesive $(\mathrm{n})$ \\
Caries-infected & $33.4 \pm 6.3(8)$ & & $7 / 1$ \\
\hline
\end{tabular}

*: significantly different $(\mathrm{p}<0.01)$

Adhesive $=$ Adhesive failure in interface

Cohesive $=$ Cohesive failure in dentin 


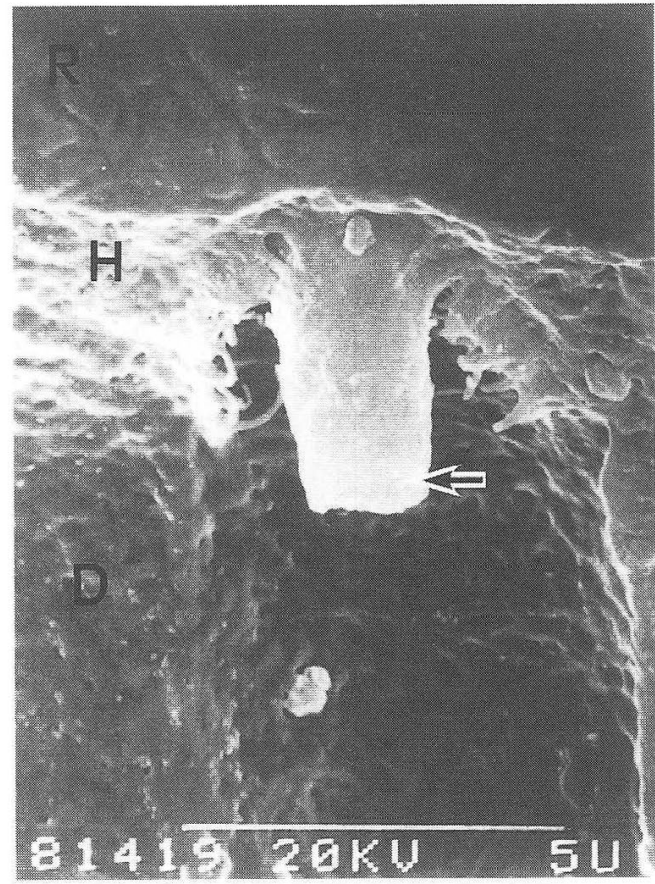

(A)

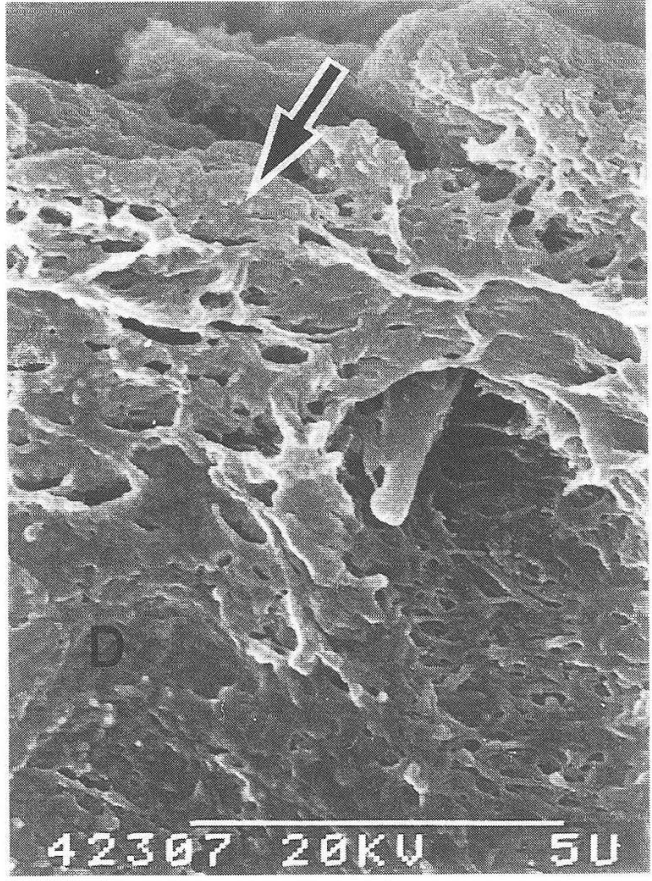

(B)

Fig. 2 SEM images of the interfaces between UB and normal vs. caries-infected dentin. ( $\Lambda$ ) Normal dentin. A short resin tag (arrow) was seen. R: bonding resin, $\mathrm{H}$ : hybrid layer, D: dentin.

(B) Caries-infected dentin. The mud-like structures (arrow) were present on the top of the caries-infected dentin.

found, so resin infiltration into the dentinal tubules of carious dentin was prevented by the mud-like structures.

The effects of UB-primer on dentin are shown in Fig. 3. (A) shows the effect of UB-primer on normal dentin; most of the smear layer and the plugs are removed. (B) shows that to carious dentin. There are bacteria-like structures in enlarged dentin tubules.

\section{DISCUSSION}

We have alreadly measured the MTBS of Liner Bond II (LB, Kuraray Co. Ltd., Osaka, Japan) and Fluoro Bond (FB, Shofu Co. Ltd., Kyoto, Japan) to human normal coronal dentin, and the MTBS of $\mathrm{LB}$ and $\mathrm{FB}$ were 28.9 and $30.5 \mathrm{MPa}$, respectively ${ }^{7)}$. The MTBS results of the present study suggested that a new self-etching/self-priming adhesive system, UB, could achieve a relatively high bond strength (more than 30 $\mathrm{MPa}$ ) to normal dentin, but that the bond strength of UB to carious dentin was significantly lower than that to normal dentin. From our SEM observation, we 


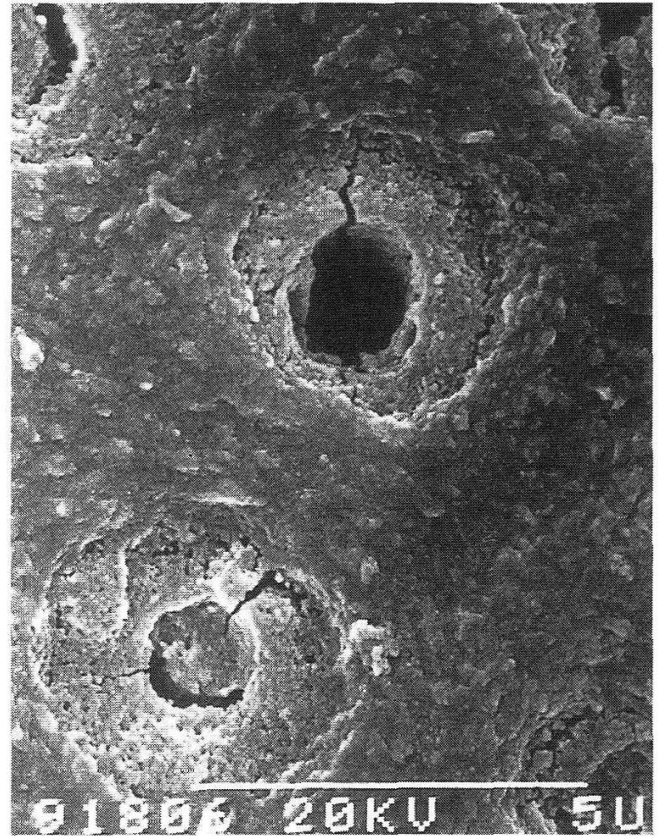

(A)

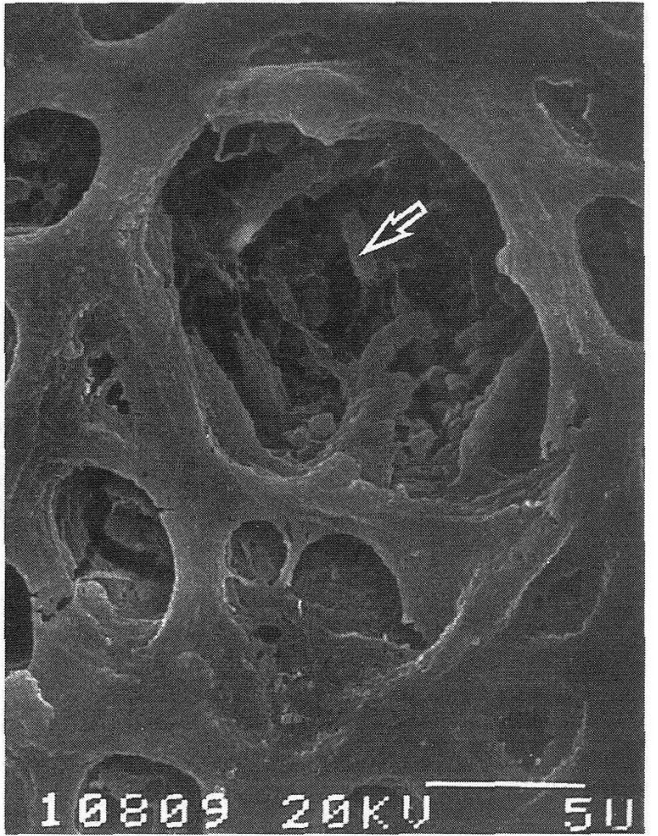

(B)

Fig. 3 SEM images of the normal and caries-infected dentin treated by UB-primer.

(A) Normal dentin. Smear layers were removed fully.

(B) Caries-infected dentin. Bacteria-like structures (arrow) were present.

speculated that mud-like structures might have inhibited the resin infiltration into the carious dentin, preventing the formation of the typical hybrid layers and resin tags. The nature of the mud-like structures is still unclear, however Sano has observed the morphology of the mud-like structures on ground carious dentin and suggested that the mud-like structures consisted of denatured carious collagen and several kinds of carious crystals $^{8)}$. Pashley et al. measured the permeability of carious dentin, and reported that the permeability of carious dentin was much less than that of normal dentin because of the occlusion of dentinal tubules with carious crystals and the presence of carious smear layers ${ }^{9)}$. Accordingly, if we want to obtain a high bond strength of UB to carious dentin, we should remove the mud-like structures and carious crystals in the dentinal tubules by use of a new pretreatment technique.

Traditional treatment of carious dentin involves removal of the carious tooth structure, preparation of the teeth to receive restorative materials, and placement of the restorative materials. The surgical and restorative concept of treatment has remained relatively unchanged from the era when Black established the traditional principles of cavity preparation, in which a great deal of dental tissue is sacrificed after caries removal ${ }^{10)}$. However, Briley et $a l$. have suggested that occlusal dental caries can be arrested for up to 10 years by resin sealing ${ }^{11}$. 
To establish a sealed restoration for dentin caries, we should evaluate the hypothesis that carious dentin can be truely arrested by resin sealing, and should develop a new pretreatment technique for resin to fully infiltrate into carious dentin.

\section{CONCLUSION}

The results of the present study have demonstrated that the MTBS of UB to cariesinfected dentin was significantly lower than that to normal dentin. SEM observation suggested that resin infiltration to caries-infected dentin would be inhibited by the presence of mud-like structures.

\section{ACKNOWLEDGEMENTS}

This study was partially supported by a Grant-in-Aid for General Scientic Research (C) (2) (No.09671956) from the Ministry of Education, Science, Sports and Culture of Japan.

\section{REFERENCES}

1) Sano, H., Shono, T., Takatsu T., Ciucchi B., Carvalho R. M. and Pashley, D. H.: Relationship between surface area for adhesion and tensile bond strength - evaluation of a micro-tensile bond test, Dent Mater 10(4) : 236-240, 1994.

2) Pashley, D. H., Sano, H., Ciucchi, B., Yoshiyama, M. and Carvalho R. M.: Adhesion testing of dentin bonding agents: A review, Dent Mater 11(2) : 117-125, 1995.

3) Nakajima, M., Sano, H., Burrow, M. F., Tagami, J., Yoshiyama, M., Ebisu, S., Ciucchi, B., Russell, C. M. and Pashley, D. H.: Tensile bond strength and SEM evaluation of caries-affected dentin using dentin adhesives, $J$ Dent Res 74(10) : 1679-1688, 1995.

4) Yoshiyama, M., Sano, H., Ebisu, S., Tagami, J., Ciucchi, B., Carvalho, R. M., Johnson, M. H. and Pashley, D. H.: Regional bond strengths of bonding agents to cervical sclerotic root dentin, $J$ Dent Res 75(6): 1404-1413, 1996.

5) Nakabayashi, N. and Pashley, D. H.: Hybridization of dental hard tissues, Quintessence Publishing Co., Ltd., Tokyo, 1998, pp.95-107.

6) Mertz-Fairhurst, E. J., Williams, J.E., Pierce, K. L., Smith, C. D., Schuster G. S., Mackert, J. R., Sherrer, J. D., Wenner, K. K., Richards, E. E., Davis, Q. B. and Ergle, J. W.: Sealed restoration: 4-year results, Am J Dent 4(1) : 43-49, 1991.

7) Yoshiyama, M., Matsuo, T., Ebisu, S. and Pashley, D. H.: Regional bond strengths of self-etching/ self-priming adhesive systems, J Dent $26: 609-616,1998$.

8) Sano, H.: Relationship between caries detector staining and structural characteristics of carious dentin, J Stomatol Soc 54(1) : 241-270, 1987.

9) Pashley, E. L., Talman, R., Horner, J. A. and Pashley, D. H.: Permeability of normal versus carious dentin, Endo Dent Traumatol $7: 207-211,1991$.

10) Black, G. V.: Operative Dentistry, Medico-Dental Publishing Co., Chicago, 1908.

11) Briley, J. B., Dove, S. B., Mertz-Fairhurst, E. J. and Hermesch, C. B.: Computer-assisted densitometric image analysis (CADIA) of previously sealed carious teeth: A pilot study, Oper Dent 22 : 105-114, 1997. 